\title{
The hammer of reason
}

\author{
In the motivation, conduct and reporting of science, there is no substitute for reason, and it must prevail whenever \\ scientific methods are used. Similarly, scientific recommendations can only be useful if they meet with rational \\ decision-making. Because people come to decisions from diverse viewpoints and values, listening to the values and \\ views of scientists and non-scientists-while explicitly refraining from debate and persuasion-may point the way to \\ determining when and where scientific ideas are of interest and likely to be adopted.
}

\begin{abstract}
$\Lambda$ grant application, report or publication is built to be an instrument of rational persuasion. As scientists, we isolate a problem, laying out our motivation, conceptual framework and tools. We test hypotheses and draw conclusions, buttressing them with converging lines of evidence, and dispense with challenges and inconsistencies by means of example, logic and demonstration. However, our conclusions are primarily absorbed and implemented by non-scientists on the basis of familiarity, acceptability and an understanding that they offer a useful solution to a perceptible problem. Only after these natural tests have been passed can most people listen to a rational argument to consider the fruits of science.

That is not however acceptance of science itself. As if the disciplinary divide were not already wide enough to prevent understanding of science, our practice of tempering reason with experience is often seen as unreasonable. The skepticism that engenders trust among scientists is often met with its mirror image: widespread public skepticism born of our claim that we know that under certain conditions our conclusions may be wrong. Further, public distrust does not only limit the promotion of new ideas and innovation: our skepticism is also incorrectly taken to imply that we distrust our own motivation and methods. This means that reason is necessary but not sufficient to generate public appreciation for experimentation with nonhuman primates, cognitive enhancement, anticancer vaccines, genetically modified crops, synthetic biology and human gene editing, to name only a few topics in our own field.

These research areas generate new possibilities so quickly that in many cases even the researchers themselves have rarely stopped to take stock of the ways their findings are changing their own priorities, options and feelings. When we interview our recent authors about the
\end{abstract}

history and trajectory of the work surrounding their recent publication, we gain a great story and a wondrous appreciation for their creativity, teamwork and motives. Debate and persuasion are laid aside because the work is already published, and we can connect with the people and their ideas. Why then would this same approach not work to generate familiarity among adversaries viewing a contentious issue from very different backgrounds and value systems? As scientists and non-scientists already come to the table with set positions, histories and views, it should be interesting to listen to both declare their conflicting interests and feelings. This could be formalized as an 'un-debate', where only personal views and intent listening matter. Attempts at persuasion, argument or rebuttal used to identify and clarify the lines on each side of the issues in debates and exchange of letters would not be appropriate in these live venues and online threads, so some degree of restraint or moderation will be called for until this type of exchange becomes familiar.

What then would be a measure of success? Realistically, we would be surprised if un-debates by themselves have the power to generate appreciation for scientific practice and ideas among those who are not already open to reason, skepticism and learning from experience. Nor would these fireside tales generate respect among scientists for those who practice unreasonable decision-making. But they would go a long way toward allowing scientists to add their human reactions and understanding of the diversity of competing issues, explanations and views outside the lab to the tools they can use to create new ideas in their work. The measure of success we would most relish is for scientists and the public alike to begin to feel comfortable that they know one another well enough as fellow humans to ask each other questions face to face-questions where a real answer matters. 